\title{
Central nervous system mycoses: The challenges
}

\author{
J. M. K. Murthy \\ Department of Neurology, The Institute of Neurological Sciences, CARE Hospital, Nampally, Hyderabad - 500 001, India
}

The incidence of central nervous system (CNS) mycoses is increasing in the last few decades mostly in the immunocompromised population. Cancer chemotherapy and solid organ and bone marrow transplantation have greatly increased the immunocompromised population. Sinocranial aspergillosis mostly described from the countries with temperate climate occurs mostly in otherwise immunocompetent individuals. ${ }^{[1]}$ The major advances in CNS mycoses are in the pathobiology, new diagnostic tools and new therapies.

The CNS pathology that the fungi evoke largely determines the clinical syndromes with which patients with CNS mycoses present. The morphology and size of the organism determine the pathology of CNS lesions. Small yeast forms (Blastomycoces, Coccidioides, Cryptococcus, Candida) reach the small arterioles and capillaries producing meningitis and subpial ischemic lesions. Intermediate-sized pseudohype (Candida) occludes small vessels in the microcirculation to produce local areas of tissue necrosis that evolve to abscess formation. The large hyphal forms (Aspergillus spp., Zygomyces spp., Cladosporium) of variable size obstruct large and intermediate-size arteries and occasionally veins, giving rise to large infarcts. In addition the immune status of the host and also the antigenic characteristics, genomic fungal DNA and on cell wall components of the pathogen also determine the pathologic lesions. ${ }^{[2]}$ The pathology in the sinocranial form of aspergillosis is a well-formed granuloma with intense fibrosis. ${ }^{[1]}$ The immunopathogenesis of CNS fungal infection is not well understood. The CNS has been considered immunologically privileged in the sense of being isolated from normal immune surveillance. Fungal infections of the CNS evoke both humoral and cellular immune response. The activation of brain resident cells combined with relative expression of immune-enhancing and immune-suppressing cytokines and chemokines may play a determinant role in the immunopathogenesis. Exploration of the genomic sequence of most fungal pathogens may help to understand the pathogenesis, virulence and immune response of host defense against these pathogens. ${ }^{[3]}$

Central nervous system mycoses require immediate and precise diagnosis and the diagnostic arsenal mainly depends on microscopy and culture of biologic samples. Culture yield may be low. Histopathological analysis can establish diagnosis of a filamentous fungal infection but cannot identify the agent at species level. Both contrast CT scan and magnetic resonance imaging (MRI) may be useful adjunctive investigations in diagnosis but cannot confirm the infection. The timing of therapeutic intervention seems to have significant impact on mortality. ${ }^{[4]}$ Early identification of patients who require antifungal therapy is therefore an important goal and requires diagnostic tools that not only have good performance characteristics, but also become positive in an early phase of the infection. In the last two decades substantial effort has been made to develop nonculture-based diagnostics. Non-culture methods include polymerase chain reaction (PCR), galactomannan (GM) antigenemia, Western blot (WB) to detect antibodies and detection of fungal metabolites D-arabinitol and $(1,3)$ beta-D-glucan. ${ }^{[5]}$ Much progress has been made in the clinical validation of these diagnostic tools for opportunistic mycoses ${ }^{[6]}$ However, there is limited data on clinical validation of these diagnostic tests in CNS mycoses. ${ }^{[7-10]}$

Central nervous system mycoses require carefully selected management approaches to optimize outcomes. Treatment outcomes in highly immunosuppressed patients remain poor. The antifungal armamentarium available to clinicians has increased in the last several years to include new formulations of amphotericin B, antifungal triazoles and the echinocandins. In recent studies voriconazole has been shown to be more effective and well tolerated in CNS aspergillosis and may be considered as first line treatment. ${ }^{[11-13]}$ The data on the efficacy of other newer antifungal agents in CNS mycoses is limited and evolving. Cost of antifungal therapy remains a crucial issue worldwide. 
Immune-based therapies for fungal CNS disease are at an exploratory level and merit further evaluation in clinical trials.

Surgery followed by aggressive systemic antifungal therapy offers the best outcomes in patients with intracranial mass lesions. ${ }^{[13]}$ Whenever feasible safe radical excision along with normal nervous tissue around it should be done. Sinocranial aspergillosis is a rare entity and the mass lesions can be intracranial extracerebral (mostly in anterior cranial fossae), intracerebral or both. These patients need surgical procedures that combine otolaryngological and neurosurgical expertise. At times radical surgical excision may not be possible and radical surgery may be associated with significant morbidity. Some of these patients may have a stormy course or rapid progression following surgery. ${ }^{[14]}$ Because of the extensive fibrosis seen in sinocranial aspergilomas ${ }^{[1]}$ therapeutic concentration of antifungal agents may not be achieved in the tissue where fungal hyphae are embedded. In patients with subtotal excision, to achieve high tissue concentration continuous intralesional administration of antifungal agents as an adjunct may be considered. ${ }^{[15]}$

It is clear from the above discussion that there are yet many challenges in the early diagnosis of CNS mycoses. However, early and precise diagnosis is the essential step to institute effective management strategies to optimize outcomes. The timing of therapeutic intervention seems to have a significant impact on mortality.

\section{References}

1. Sundaram C, Umabala P, Laxmi V, Purohit AK, Prasad VS, Panigrahi M, et al. Histopathology of fungal infections of central nervous system: A seventeen years experience from south India. Histopathology 2006;49:396-405.

2. Shankar SK, Mahadevan A, Sundaram C, Saraker C, Chacko G, Lanjewar DN, et al. Pathobiology of fungal infections of the central nervous system with special reference to the Indian scenario. Neurol India 2007;55:198-215.

3. Dotis J, Roilides E. Immunopathogenesis of central nervous system fungal infections. Neurol India 2007;55:215-20.

4. Morrell M, Fraser VJ, Kollef MH. Delaying the empiric treatment of Candida bloodstream infection until positive blood culture results are obtained: A potential risk factor for hospital mortality. Antimicrob Agents Chemother 2005;49:3640-5.

5. Reiss E, Obayashi T, Orle K, Yoshida M, Zancope-Oliveira RM. Non culture based diagnostic tests for mycotic infections. Med Mycol 2000;38:147-59.

6. Mennink-Kersten MA, Verweij PE. Non culture-based diagnosties for opportunistic fungi. Infect Dis Clin North Am 2006;20:711-27.

7. Kami M, Ogawa S, Kanda Y, Tanaka Y, Machida U, Matsumura T, et al. Early diagnosis of central nervous system aspergillosis using polymerase chain reaction, latex agglutination test and enzyme-linked immunosorbent assay. Br J Haematol 1999;106:536-7.

8. de Almeida SM, Queiroz-Telles F, Doi EM, Ono M, Werneck LC. Antigp43 antibodies in the cerebrospinal fluid of patients with central nervous system involvement by paracoccidiodomycosis. Am J Clin Pathol $2002 ; 118: 864-8$.

9. Viscoli C, Machetti M, Gaxxola P, Maria A, Paola D, Van Lint MT, et al. Aspergillus galaactomannan antigen in the cerebrospinal fluid of bone marrow transplant recipients with voriconazole. J Clin Microbiol 2002;40:1496-9.

10. Machetti M, Zotti M, Veroni L, Mordini N, Van Lint M'T, Bacigalupo A, et al. Antigen detection in the diagnosis and management of patient with probable cerebral aspergillosis treated with voriconazole. Transpl Infect Dis 2000;2:140-4.

11. Stevens DA, Kan VL, Judson MA, Morrison VA, Dummer S, Denning DW, et al. Practice guidelinesfor disease caused Aspergillus. Clin Infect Dis 2000;30:696-709.

12. Herbrecht R, Denning DW, Patterson TE, Bennet JE, Greene RE, Oestmann JW, et al. Voriconazole versus amphotericin B for primary therapy of invasive aspergillosis. N Engl J Med 2002;347:408-15.

13. Schwartz S, Ruhuke M, Riband P, Corey L, Driscoll T, Cornely OA, et al. Improved outcome in central nervous system aspergillosis using variconazole treatment. Blood 2005;106:2641-5.

14. Siddiqui AA, Shah AA, Bashir SH. Craniocerebral aspergillosis of sino-nasal origin in immunocompetent patients: Clinical spectrum and outcome in 25 cases. Neurosurgery 2004;55:602-13.

15. Camarata PJ, Dann DL, Falney AC, Parker RG, Seljeskog EL. Continual intracavitary administration of amphotericin $B$ as an adjunct in the treatment of Aspergi llus brain abscess: Case report and review of the literature. Neurosurgery 1992;31:575-9. 